\title{
THE UPGRADED RF SYSTEM FOR THE AGS AND HIGH INTENSITY PROTON BEAMS*
}

\author{
J.M. Brennan \\ Conf $9505 / 2--50$ \\ AGS Department, Brookhaven National Laboratory \\ Upton, New York 11973-5000 USA
}

\section{Abstract}

The AGS has been upgraded over the past three years to produce a record beam intensity of $6 \times 10^{13}$ protons per pulse for the fixed-target physics program. The major elements of the upgrade are: the new $1.5 \mathrm{GeV}$ Booster synchrotron, the main magnet power supply, a high frequency longitudinal dilution cavity, a feedback damper for transverse instabilities, a fast gamma transition jump system, and a new high-power rf system. The new rf system and its role in achieving the high intensity goal are the subjects of this report.

The if system is heavily beam loaded, with 7 Amps of $\mathrm{rf}$ current in the beam and a peak power of $0.75 \mathrm{MW}$ delivered to the beam by ten cavities. As an example of the scale of beam loading, at one point in the acceleration cycle the cavities are operated at $1.5 \mathrm{kV} /$ gap; whereas, were it not for the new power amplifiers, the beam-induced voltage on the cavities would be over $25 \mathrm{kV} /$ gap. The upgraded if system, comprising: new power amplifiers, wide band rf feedback, improved cavities, and new low-level beam control electronics, is described. Results of measurements with beam, which characterize the system's performance, are presented. A typical high intensity acceleration cycle is described with emphasis on the key challenges of beam loading.

\section{CAVITY}

Figure 1 shows a schematic drawing of one of the ten cavity stations, comprising four ferrite-loaded(4L2) push-pull cells connected in parallel. Each cell provides up to $10 \mathrm{kV}$. The ferrite bias current, 0 to $1500 \mathrm{~A}$, flows in the walls and interconnecting "bus bars". They operate between 1.6 and 4.5 MHz. The range for protons is 2.7 to $3.0 \mathrm{MHz}$, but the full range is needed for heavy ions. The upgrade program did not need to substantially change the cavities, however, two important improvements were made.

\section{$R / Q$}

With batch filling from the Booster, the AGS must operate with a partially filled ring at high intensity and is subject to transient beam loading. The best cure for transient beam loading is to reduce the R/Q of the cavity. For these cavities

*Work performed under the auspices of the U.S. Department of Energy.

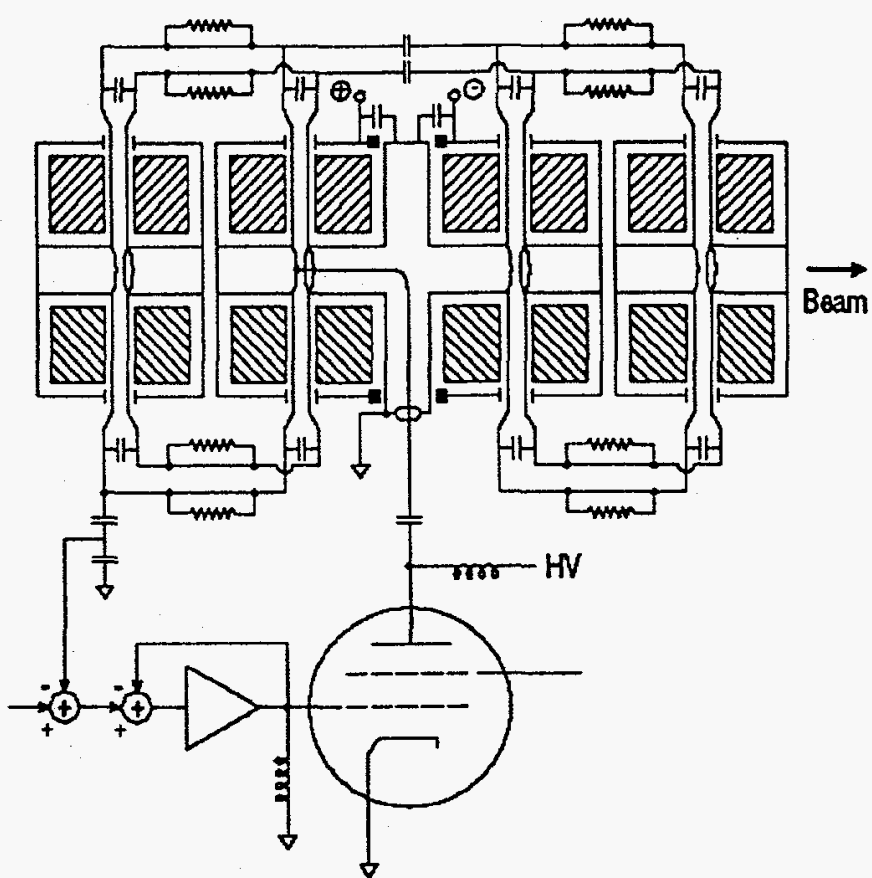

Figure 1. The four-cell cavity with, tetrode power amplifier, feedback amplifier, split loading capacitors at the gaps, and damping resistors on the bus bars.

the $R / Q$ is easily controlled by the external capacitors loading the gaps. For the upgrade the capacitance was increased as much as possible. The limit is given by the ferrites. As the capacitance increases, the biasing of the ferrites must increase. However, the dissipation in the ferrites increases with bias but must be below $300 \mathrm{~mW} / \mathrm{cm}^{3}$. In addition, the ferrite exhibits a Q-loss instability at the top frequency which also depends on bias. The capacitors were increased from $275 \mathrm{pF}$ to $600 \mathrm{pF}$. The intrinsic capacitance of the structure is 130 $\mathrm{pF}$. Therefore, the $\mathrm{R} / \mathrm{Q}$ was decreased by a factor of 1.8 to a value of $80 \Omega$ at midband.

\section{Higher Order Modes}

Larger loading capacitors brought down the frequencies of resonant modes on the "bus bars" connecting the cells. These higher order modes interfere with the If feedback and cause narrowband impedances capable of driving coupled-bunch longitudinal beam instabilities. Damping of these modes was facilitated by existing extra leadouts from the gaps, on the left side of the beam tube as well as the right. By adding "bus 
bars" on the left side, in parallel with those on the right, and splitting the loading capacitors to $300 \mathrm{pF}$ on each side, the frequencies of the higher order modes were increased. Then, the modes were effectively damped by resistors in parallel with all the "bus bars". The resistors are $40 \Omega, 25 \mathrm{~mm}$ by $600 \mathrm{~mm}$ low-inductance power resistors.

\section{POWER AMPLIFIER}

\section{Power Tetrode}

The power amplifier uses a Thomson-CSF TH573 $300 \mathrm{~kW}$ tetrode in the grounded-cathode configuration. The anode power supply can deliver an input power of $390 \mathrm{~kW}$. The tube must provide $60 \mathrm{~kW}$ to drive the four-cell cavity to 40 $\mathrm{kV}$, and provide up to $75 \mathrm{~kW}$ of beam power at $6 \times 10^{13} \mathrm{ppp}$. There are ten amplifier/cavities in the system. The surplus installed power provides the opportunity for future intensity upgrades but also is instrumental for achieving stability under the heavily beam-loaded conditions. The choice of a tetrode is attractive because it leads straightforwardly to a stable amplifier. The high power tetrode has the additional feature of a relatively low anode resistance which significantly loads the cavity, thereby reducing its impedance. This property is an integral part of if system's design, as it yields a factor of three or more of broadband impedance reduction. A beneficial aspect of this type of impedance reduction is that it can be dynamically controlled by changing the grid bias of the tube. In this way the loading effect can be enhanced at key points in the acceleration cycle and then reduced, to conserve power, when not necessary. Typically the amplifier operates in class $A B 1$, but is put into class $A$ at peak beam loading times and is biased to cut-off when the if system is off (roughly $60 \%$ of the repetition period).

\section{Reactive Power}

At times the power amplifier must drive a de-tuned load. Transients occur at injection, transition, and de-bunching (at the unstable fixed point) before the slow extraction. The cavity tuning servos require about $3 \mathrm{~ms}$ to settle to the new compensation current. To maintain constant cavity voltage during the settling time, the power amplifiers must deliver reactive current. As much as $50 \mathrm{~A}$ average reactive current could be called for at transition. In this case the power tetrode would reach 180 A peak during the if cycle.

\section{Coupling to the Cavity}

To fully exploit the low plate resistance of the power tube, it must couple to the gap without impedance transformation. Further, the line must be short to prevent standing wave modes at low frequency. Also, wideband rf feedback requires a minimum of delay in the feedback path. Figure 1 shows the coupling line between tube and gap, which is $1.5 \mathrm{~m}$ long. The line crosses a gap and the current returns to ground by encircling the ferrite of both halves of the cavity cell. The single-ended voltage on the anode appears as an equal magnitude push-pull voltage on the gap, hence the impedance ratio is 1:1. Referred to a single gap, however, the tube impedance is effectively quadrupled because the four cells are in parallel. The line travels inside the beam tube in the vacuum, eliminating potential high voltage breakdown problems. The beam is shielded from the line by a grounded inner sleeve. To install the coupling line, the cavities were disassembled into individual cells. This provided an opportunity to upgrade all the vacuum seals of the cavities. Fortunately, neither the ceramic insulators at the gaps nor the ferrite stacks had to be disassembled. The coupling line is water cooled with approximately $2 \mathrm{gpm}$ of flow.

\section{RF FEEDBACK}

At injection the cavities are operated at $1.5 \mathrm{kV} / \mathrm{gap}$, which requires $0.5 \mathrm{~A}$ of current from the power amplifier, $\left(\mathrm{L}_{0}\right)$. At $6 \times 10^{13}$ ppp the rf beam current, $\left(I_{B}\right)$, is $6.0 \mathrm{~A}$, implying a beam loading parameter, $I_{B} / I_{0}$, of 12 . It has been shown $[1,2]$ that when the beam loading parameter becomes greater than 2 the beam control loops, tuning, AVC, and phase, are crosscoupled and become unstable. RF feedback is needed to reduce the effective beam loading parameter. Feedback reduces the perturbations of the gap voltage by the value of the loop gain, and the beam current, seen from the control loops, is effectively reduced. Loop gains of $17 \mathrm{~dB}$ and greater (depending on the operating point of the tetrode) are used to reduce the beam loading parameter to less than 1.7.

Other peripheral benefits follow from rf feedback: 1 . cavity control is linearized, the AVC loop does little; 2. phasing of the cavities is essentially independent of tuning servo errors; and 3. the modulation response time of the cavities is reduced by the loop gain. The hardware of the rf feedback is described in some detail elsewhere. $[3,4]$ Some key points deserve emphasis. The feedback amplifier is a closed-loop circuit itself. It must have wide bandwidth to add minimal phase shift in the cavity loop. For phase margins less than $60^{\circ}$ the closed loop response will exhibit gain peaking which, increases the cavity impedance at adjacent revolution harmonics. Unstable coupled-bunch modes could be driven by the peaking. Closed loop operation of the feedback amplifier adds negligible delay to the cavity loop, and phase margins greater than $60^{\circ}$ are maintained. The key component for achieving the wide bandwidth is the attenuator that senses the grid voltage. The load resistance on the grid is set to $200 \mathrm{Q}$. This is a compromise between a low value to keep the $Q$ of the tuned circuit low and a high value to limit the required current from the amplifier. Attempts to transform a standard $50 \Omega$ load to $200 \Omega$ resulted in spurious resonances that destabilized the loop. A better solution was to obtain a special (Altronics Research Inc.), water-cooled, $200 \Omega$ 10:1 attenuator which gave a clean spectrum to very high frequencies. Finally, the control grid of the tetrode constitutes a significant capacitive load $(\sim \operatorname{lnF})$ which must be driven to 300 Volts. 


\section{DISCLAIMER}

Portions of this document may be illegible in electronic image products. Images are produced from the best available original document. 


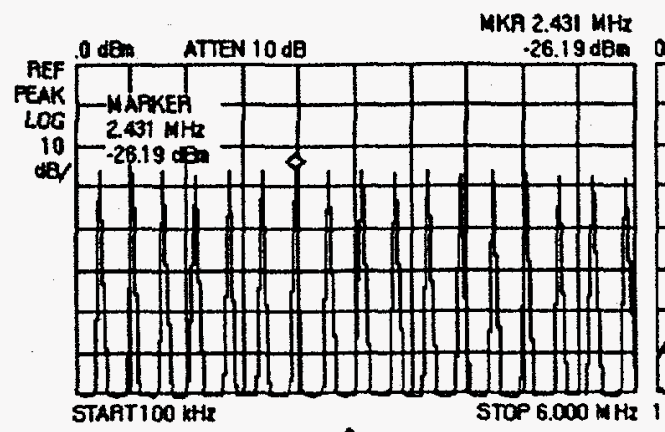

2.

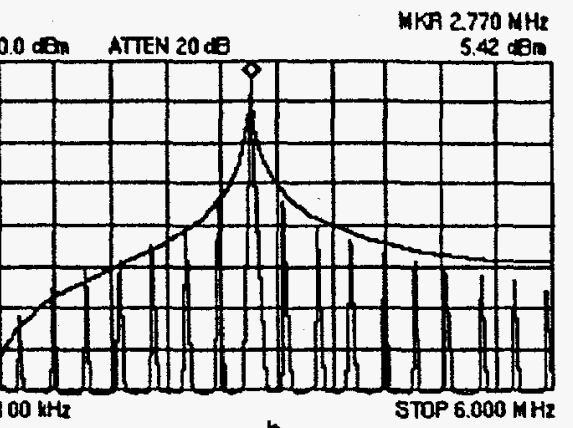

b.

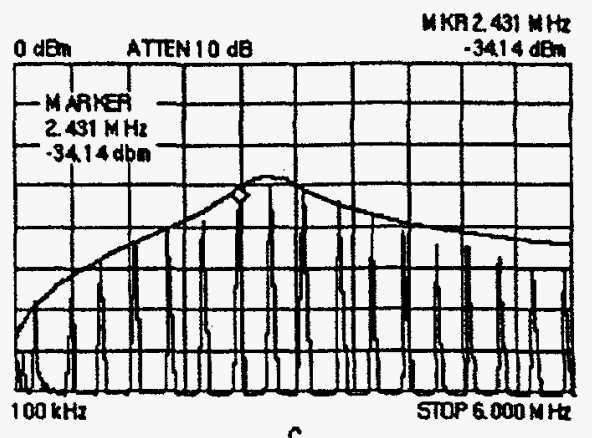

c.

Figure 2. Cavity impedance measurements; a. beam current, b. cavity voltage with amplifier off, c. cavity voltage with amplifier and if feedback on.

By resonating the grid capacitance with a variable inductor, the size of the feedback amplifier is kept modest. The inductor is a biased ferrite (4M2). Bias current is derived from a measurement of the operating frequency.

\section{Protection Systems}

Six systems prevent damage from over-power situations. 1. A fast crowbar circuit on the tetrode anode voltage protects against arcs within the tube or sudden loss of grid bias. 2. A slow overload circuit shuts down the anode power supply at $35 \mathrm{~A}$ average current. 3 . If the rf voltage at the control grid exceeds $300 \mathrm{~V}$, the drive signal to the power amplifier is shut down. 4. A window comparator about the reference signal for the AVC loop checks for out-of-range situations, such as, a spark in the cavity. This circuit inhibits the power amplifier drive. 5. The feedback amplifier is equipped with a input voltage limiter made from PIN diodes. The limiter would act in the event that the feedback signal from the cavity failed. 6 . The bias current for the ferrites of the cavity and grid resonator are monitored and interlock the If drive on a fault.

\section{MEASUREMENTS WITH BEAM}

The beam provides a pure current source and makes a definitive probe of the cavity impedance. By storing the beam with nine of the cavities, the voltage induced on a test cavity was measured. Three aspects of the cavity impedance have been measured: 1 . the transient response to a single bunch yields the $R / Q, 2$. the impedance at the fundamental if component yields the cavity and power-tube resistance, 3 . the shape of the impedance shows the effect of if feedback.

$R / Q$

A single bunch of $2 \times 10^{12}$ protons, 120 ns wide was injected into the ring and the induced voltage on a cavity was observed. From the response of the cavity to the first bunch passage, an $R / Q$ of $81 \Omega$ was obtained. This is consistent with the size of loading capacitors, $600 \mathrm{pF}$.

\section{RF Feedback}

RF feedback does not reduce the impedance of the cavity but it does reduce the impedance that the beam "sees". It also reduces the beam intensity that the beam control loops "see". To measure the effectiveness of the feedback the cavity was stimulated with beam of wide spectral content by using a single bunch, kept short by the other cavities. Figure 2a shows the spectrum of the beam current. The short bunch makes spectral lines of almost constant amplitude over the first 17 revolution harmonics. The cavity is tuned to the eighth harmonic with the power amplifier off and shows a characteristic resonance response with a $Q$ of 50 in Figure $2 b$. In Figure $2 c$ the power amplifier and the if feedback have reduced the $Q$ to 5 , which is consistent with the feedback loop gain. These results agree well with low-level network analyzer measurements but are more rigorous in that they stimulate the cavity directly at the gap with beam and are done at high level. At $25 \mathrm{MHz}$, the beam signal is down by $20 \mathrm{~dB}$ but is still useful in showing that the higher order modes on the busbars have been effectively damped.

\section{Tetrode Output Resistance}

The output resistance of the power tube can be varied over a wide range by changing the grid bias of the tube. The output resistance was measured as a function of plate current with a full complement of bunches in the ring. Two spectrum analyzers, triggered simultaneously, measured the beam from a longitudinal pick-up and the cavity voltage. By measuring with the tetrode switched off, the resistance of bare cavity was determined. Three precautions were necessary to insure that only the tube resistance changed as the tube current was varied: 1 . the cavity was kept on resonance by locking the tuning servo to the beam, 2 . incidental negative feedback around the tube caused by plate-to-grid capacitance was negated by switching the input of the feedback amplifier to ground, 3. the level-dependence of the ferrite loss was avoided by making measurements at constant gap voltage by varying the beam current. Figure 3 shows the results as function of tube current. Measured conductances are plotted, 
along with values obtained from manufacturer's tube characteristics curves. The agreement is good. These results demonstrate that the beam loading capability of the if system can be boosted by a factor of three at key points in the cycle by changing the operating point of tube.

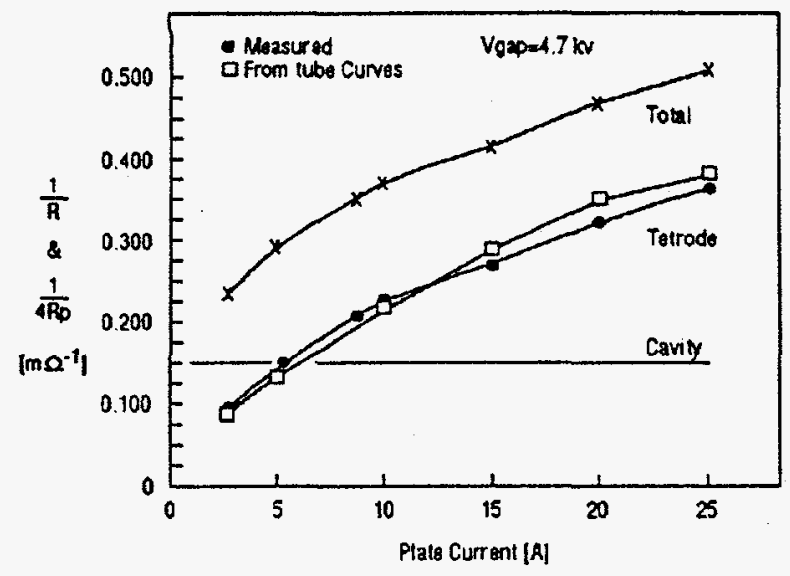

Figure 3. Output conductance of the power tetrode versus average plate current. Squares are from tube characteristics.

\section{HIGH INTENSITY OPERATION}

A comprehensive discussion of the challenges of high intensity operation is given elsewhere in these proceedings. [5] The discussion here focuses on the issue of beam loading. The critical points in the cycle for beam loading are at: injection and accumulation, gamma transition, and debunching before the slow beam extraction. Figure 4 shows some key signals of the high intensity proton operating cycle. One can see that at the critical points the rf voltage is low, aggravating the beam loading effect. At transition and debunching the tube current is momentarily bumped up.

\section{Injection and Accumulation}

The Booster supplies two bunches of $1.5 \mathrm{GeV}$ (kinetic) protons four times at $133 \mathrm{~ms}$ intervals. At high intensity it is essential to keep the bunches as long as possible to minimize space charge tune shifts. This implies that the rf voltage be low, but not zero, so that the beam stays bunched to provide clear space on the ring for the incoming beam. The voltage is limited on the low side by the need for enough bucket height to cover the Booster beam momentum spread. How the bunches become long (roughly double in length) is an important matter. Simple filamentation in a mismatched bucket leads to "hot spots" in the bunch distribution. Furthermore, each batch of bunches has a different history with the first batch waiting $400 \mathrm{~ms}$ for the last to arrive. While at transition one wants the eight bunch distributions to be as equal as possible. An additional constraint on the possibilities at injection comes from the fast transition jump system. In perturbing the machine lattice it multiplies the dispersion function by a factor of four and constricts the momeatum aperture of the machine. This constriction limits the maximum longitudinal emittance before transition and implies low rf voltage must be used to make long bunches. In the fixed-target, slowlyextracted-beam mode there is no premium on low emittance. In fact, after transition the emittance is intentionally blown up by a factor of three before extraction.

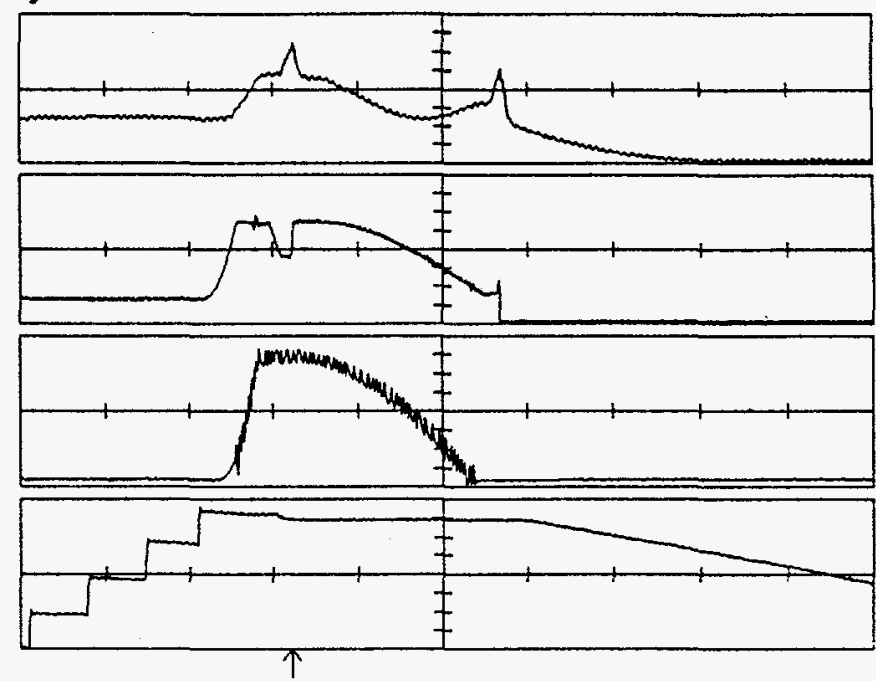

Figure 4. High intensity proton operation. From bottom: beam intensity, main magnet field derivative, net if voltage, and typical power amplifier average current. Arrow at bottom indicates transition. Time axis is $2 \mathrm{~s}$ full scale.

The injection and accumulation phase is the principal challenge of high intensity operation. The following strategy has evolved. The if is operated at low voltage, 1.5 to 2 $\mathrm{kV} /$ gap ( $x 40$ gaps). The bunches are injected off-center in the bucket, causing a dipole synchrotron oscillation, mixed with quadruple oscillation from the low voltage. A high frequency $\left(33 \mathrm{xf}_{\mathrm{sf}}\right.$ ) "dilution" cavity supplies $20 \mathrm{kV}$ for $50 \mathrm{~ms}$ after each injection. The local non-linearities from the high frequency smooth out the bunch distribution and eliminate the hot spots[6]. To prevent the dipole oscillation of the new batch from imposing a new dipole oscillation on the old batches via the phase loop (average phase), and driving additional dilution, the new batch is gated out of the phase measurement, turn-by-turn, until the dipole signal has damped to zero from filamentation and dilution.

\section{Transition}

Because of the dispersion blow-up caused by the transition jump system the if voltage is dropped to the point where the bucket area equals the emittance while the jump system operates. This minimizes the beam momentum spread and is essential for controlling losses. From Figure 4 one can see that the beam loss occurs before the actual transition, while the jump quadrupoles are ramping up.

The power amplifier must drive a de-tuned (anti-compensated) cavity for 3 to $5 \mathrm{~ms}$ after the transition phase jump until the tuning servo settles to compensate the reactive beam 
current. For the cavity voltage to remain stable the amplifier must supply $2 \mathrm{I}_{\mathrm{B}} \operatorname{Cos}\left(\varphi_{\mathrm{g}}\right) \times(4 \mathrm{gaps})$ of reactive current. At 6 $x 10^{13} \mathrm{ppp}$, and synchronous phase angle, $\varphi_{1}=63^{\circ}$, this is 25 A. While the real part of the current is $35 \mathrm{~A}$. A vector diagram, following the conventions of Pedersen [1], of the currents at transition is shown in Figure 5. As shown in Figure 4 the average tube current has been bumped up to 23 A dc for $20 \mathrm{~ms}$ at transition. The benefits of the power boost are, lower plate resistance, as discussed above, and higher transconductance of the tube which increases if feedback loop gain. The currents at this intensity are well within the capability of the tetrode.

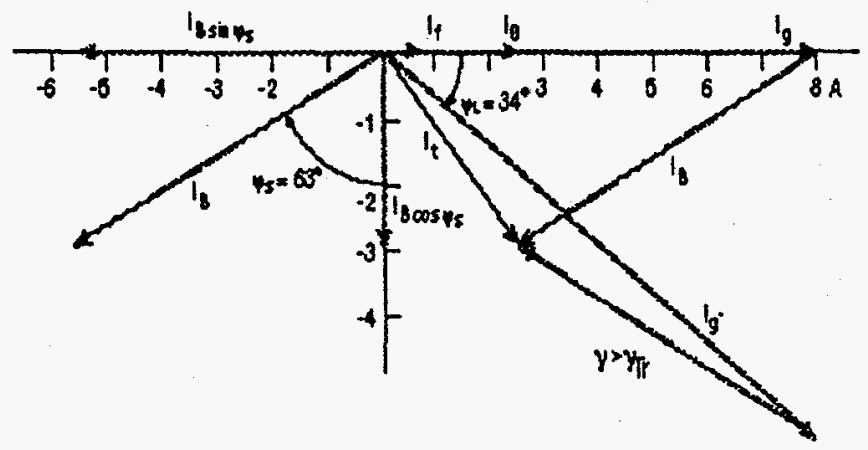

Figure 5. Phasor diagram of currents at transition. The voltage is horizontal to the right. $\mathrm{I}_{0}$ is current with zero beam. $I_{B}$ is beam current, which flips at transition. $I_{B}$ is tube current.

\section{Duck Under Transition}

The diagram of Figure 5 illustrates that a larger synchronous phase angle leads to less reactive current. Ultimately, at $90^{\circ}$ there is zero reactive current. This fact was actually exploited in the first year's run with the new power amplifier. A problem with the dc power supply for the anode voltage, limited the available voltage and current to the extent that insufficient reactive current was produced[4]. To get smoothly through transition the synchronous phase was raised to $90^{\circ}$ at transition by dropping the if voltage, then returned to the normal value in about $5 \mathrm{~ms}$. The tuning servo can track this rate, so the cavity remains compensated at all times. This is the old scheme of "ducking under transition"[7], which was proposed as a cure for transition problems before the transition jump scheme came into use. The problem with ducking under transition is that at $90^{\circ}$ there is zero bucket area and all the beam is unstable. It never really solved the transition problem but it does have this spin-off benefit for the rf system. However, when the transition jump is used one never really gets too close to transition and although distortions may occur as the bucket collapses, they tend to reverse after the jump when the voltage is raised again. Our experience has been that no beam loss is incurred by using the duck-under, but the benefit to the power amplifier can be important. This maneuver could be useful again in the AGS at higher intensities.

\section{De-bunching for Slow Extraction}

The most severe moment of beam loading is at de-bunching for slow extraction. The power boost indicated in the top trace of Figure 4 is used bere. The rf voltage is low and the beam phase is flipped $180^{\circ}$ to the unstable fixed point to drive up the momentum spread and cause de-bunching. The if voltage is dropped by a factor of three because this produces the flattopped momentum distribution needed for a constant extraction rate over $1.2 \mathrm{~s}$. The combination of low rf voltage and a fast $180^{\circ}$ phase jump taxes the power amplifier/cavity system. All the beam current is reactive so the amplifier must supply over 50 A while the tuning servo changes the impedance angle by $160^{\circ}$.

A much less demanding situation occurs if the net voltage reduction is obtained by counter phasing the cavities in pairs to $144^{\circ}$. The individual cavity voltage is not reduced so $I_{0}$ stays high (the ferrite resistance is also lower by a factor of two). Most importantly, there is zero reactive current called for. There is real current of opposite sign and different magnitude between the cavity pairs, but the magnitude is less than the reactive current without counter phasing. Before the phase flip all the cavities are compensated by the tuning servos with the same impedance angle. When the net cavity phase jumps $180^{\circ}$, the counter-phased cavities just change roles. The signs and magnitudes of the real currents interchange but the tuning servo is not perturbed, since it feeds back on the reactive current.

\section{CONCLUSION}

The new high power if system for the AGS has been completed and accelerates a record intensity of $6 \times 10^{13}$ protons per pulse. Measurements with beam of the impedance of the cavity-power amplifier system have been carried out. The capability to accommodate heavy beam loading has been demonstrated and indicate that the intensity goal of $7 \times 10^{13}$ protons per pulse for 1996 is realistic.

\section{REFERENCES}

[1] F.Pedersen, IEEE Trans. Nuc. Sci., NS-22,1975,p1906. [2] D. Boussard, Proc. CERN School on RF Engineering, Oxford, England, April 1991.

[3] J.M.Brennan, et al. Proc. of the 1993 IEEE Particle Accelerator Conference, Vol 2,p.1241, Washington DC.

[4] J.M.Brennan, et al. EPAC June 1994, Vol. 3, p.1897, London England.

[5] M.Blaskiewicz, et al. these proceedings.

[6] R. Cappi, et al. Proc. of the 1993 IEEE Particle Accelerator Conference, Vol 5, p.3570, Washington DC.

[7] A. Sorenssen, Particle Accelerators, 1975. Vol 6. p.141. 


\section{DISCLAIMER}

This report was prepared as an account of work sponsored by an agency of the United States Government. Neither the United States Government nor any agency thereof, nor any of their employees, makes any warranty, express or implied, or assumes any legal liability or responsibility for the accuracy, completeness, or usefulness of any information, apparatus, product, or process disclosed, or represents that its use would not infringe privately owned rights. Reference herein to any specific commercial product, process, or service by trade name, trademark, manufacturer, or otherwise does not necessarily constitute or imply its endorsement, recommendation, or favoring by the United States Government or any agency thereof. The views and opinions of authors expressed herein do not necessarily state or reflect those of the United States Government or any agency thereof. 